\title{
El Trauma del Nacimiento - I \\ Las alteraciones del sueño en el niño con alto riesgo perinatal
}

Dr. Jaime Quevedo Caicedo

\section{INTRODUCCION}

Poco es lo que se conoce sobre el significado clínico de los trastornos del Sueño en los niños. Se conoce que son frecuentes entre el segundo y tercer años de vida; se conocen también los requisitos en los tres primeros años de vida y es amplia la bibliografía sobre los mecanismos y funciones del Sueño en adultos. (1) (2) (3). En el análisis del seguimiento de NARP se evidenció que esta alteración se presentaba en el $46 \%$ de los niños y en el estudio comparativo con niños SIN riesgo perinatal se demostró que el trastorno se presenta en el $66.25 \%$ del grupo testigo en comparación con un $7.89 \%$ en el grupo control. De este estudio se pudo concluir que hay una relación altamente significativa entre los trastornos del sueño y el riesgo perinatal, lo cual permitirá colegir que puede existir una base orgánica que determine este problema. El riesgo relativo encontrado fué de $8 \times 1$, lo cual quiere decir que de cada ocho niños con Tr. del S. solo uno tiene causas distintas del riesgo perinatal. Estas consideraciones nos obligaron a darles un capítulo especial.

\section{MATERIAL Y METODOS}

Del mismo grupo de NARP que habían cumplido 20 o mas meses de seguimiento se seleccionaron todos los niños que habían presentado Trastornos del Sueño en algún momento de su seguimiento. Se encontraron 73 niños, de los cuales 48 eran varones y 25 mujeres. La relación hombre-mujer es de casi $2 \times 1$ lo cual es un promedio muy protuberante para el sexo masculino.

\section{RESULTADOS}

En el cuadro No. 1 se muestra la distribución según la edad de aparición del Trastorno; se observa que el $75 \%$ de los niños empiezan a presentar el problema antes del $8 \&$ mes de la vida y el $25 \%$ restante entre los 9 meses y dos años.

La sistomatológica del Trastorno se resume

\begin{tabular}{|lccccc|}
\hline \multicolumn{5}{|c|}{ LA ALTERACION DEL SUEÑO EN EL NIÑO DE ALTO RIESGO } \\
\hline I. S. S. - Caja Seccional del Valle - Cali & MUJERES & TOTAL \\
\hline EDAD & VARONES & No. & $(\%)$ & \\
\hline $0-4$ Sem. & No. & $(\%)$ & 5 & $(6.84)$ & 20 \\
$0-4$ Sem. & 15 & $(20.5)$ & 3 & $(4.10)$ & 14 \\
$1-3$ Mes. & 11 & $(15.06)$ & 11 & $(15.06)$ & 21 \\
$4-8$ Mes. & 10 & $(13.7)$ & 2 & $(2.73)$ & 9 \\
$9-12$ Mes. & 7 & $(9.58)$ & 4 & $(5.48)$ & 9 \\
-1 Año & 5 & $(6.84$ & 25 & $(34.24)$ & 73 \\
\hline
\end{tabular}




\begin{tabular}{|c|c|c|c|}
\hline & CARASTEROSTOCAS CLINICAS & No. & $(\%)$ \\
\hline & CARACTERISTICAS CLINICAS & No. & $(\%)$ \\
\hline 1. & Se despierta varias veces en la noche. & 39 & $(53.42)$ \\
\hline 2. & $\begin{array}{l}\text { Se despierta con gritos, alaridos anormales, } \\
\text { llanto angustioso. }\end{array}$ & 33 & $(45.20)$ \\
\hline 3. & $\begin{array}{l}\text { Presenta sobresaltos severos "como si súbi- } \\
\text { tamente quedara en el vacio". }\end{array}$ & 39 & $(53.42)$ \\
\hline 4. & Irritabilidad nocturna excesiva. & 16 & $(21.9)$ \\
\hline 5. & Dificultad para conciliar el sueño. & 7 & $(9.6)$ \\
\hline 6. & $\begin{array}{l}\text { Duerme muy poco, se acuesta tarde, se des- } \\
\text { pierta muy temprano. }\end{array}$ & 6 & $(8.2)$ \\
\hline 7. & Temblor o clonías de extremidades. & 20 & $(27.4)$ \\
\hline 8. & Solo se anotó Alt. Severa del Sueño & 24 & $(32.9)$ \\
\hline
\end{tabular}

en el cuadro No. 2. Se aprecia que el compromiso se establece tanto para el ritmo y la duración de los períodos de sueño como para las características del mismo.

\begin{tabular}{|lrc|}
\hline $\begin{array}{l}\text { TIEMPO DE } \\
\text { DURACION }\end{array}$ & No. & $\%$ \\
\hline $0-2$ meses & 10 & $(13.7)$ \\
$3-6$ meses & 12 & $(16.4)$ \\
7 -12 meses & 8 & $(10.9)$ \\
-1 año & 25 & $(34.2)$ \\
Aparición esporádica & 18 & $(24.6)$ \\
& 73 & $(100.0)$ \\
\hline & & \\
CUADRO No. 3 & & \\
\hline
\end{tabular}

Én el cuadro No. 3 se muestra la distribución según la duración del Trastorno; se aprecia una distribución variable que indica que el trastorno puede tener una duración impredecible y que puede aparecer por intervalos inespecíficos.
En el cuadro No. 4 se muestra la distribución de las convulsiones en este grupo de niños. El que un $56.1 \%$ de estos niños presenten episodios convulsivos confirma -en cierta forma - la probable causa orgánica del trastorno. El que no colvulsionen un $43.8 \%$ no está en contra de esta afirmación; creemos que la maduración cerebral es un proceso dinámico̊ muy fuerte que tiende a recuperar en grado no calculado los daños causados por la lesión cerebral perinatal. Las características de la convulsión son similares a las descritas en el capítulo anterior.

En el cuadro No. 5 se muestra la distribución de la relación de tiempo entre la aparición de la alteración del sueño y el episodio convulsivo. Se observa que el Trastorno del Sueño precede el episodio convulsivo -en el $65.8 \%$ de los casos- por períodos que van desde 4 meses a 1 año; en el $14.6 \%$ el trastorno es concomitante con la convulsión y en un $19.5 \%$ éste continua como una complicación adicional postconvulsiva.

Como dato simplemente informativo, que 


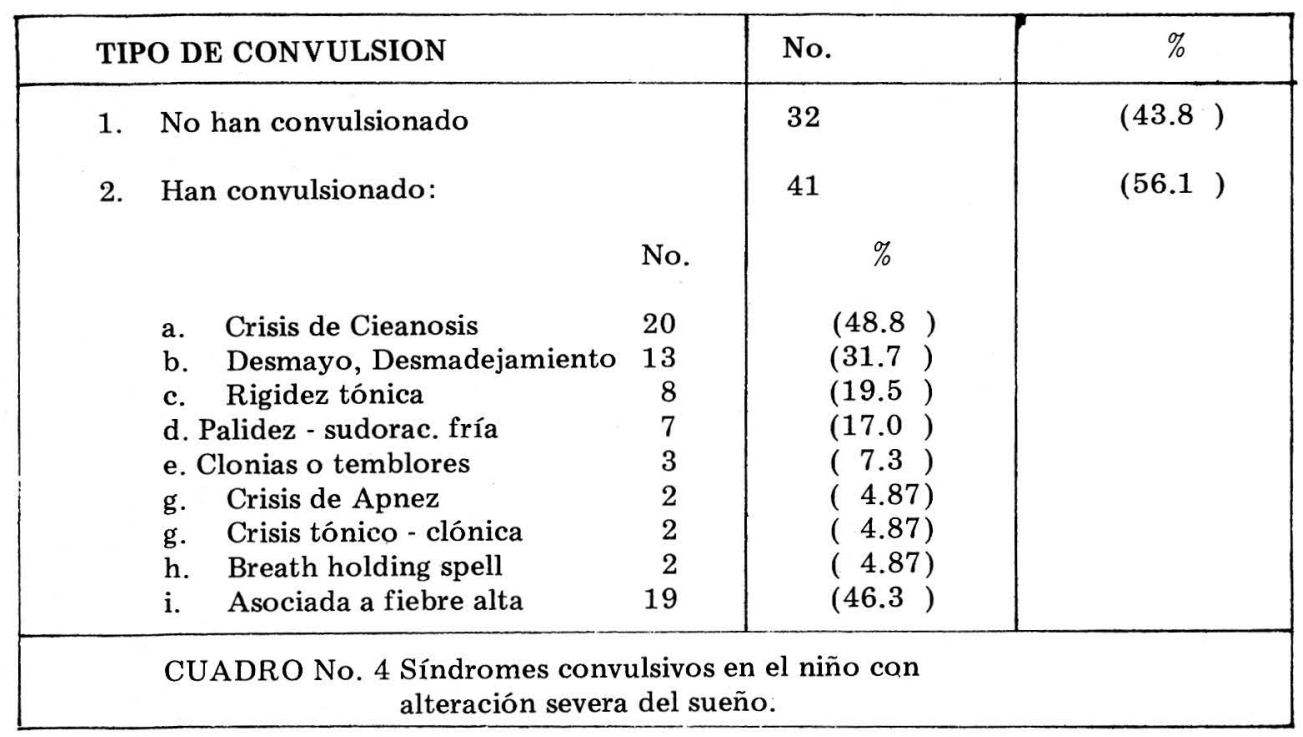

\begin{tabular}{|lll|}
\hline INTERVALO & No. & $\%$ \\
\hline $0-3$ meses & 0 & $(9.75)$ \\
$0-3$ meses & 0 & \\
$4-6$ meses & 4 & $(9.75)$ \\
7 -12 meses & 4 & $(9.75)$ \\
-1 año & 19 & $(46.34)$ \\
Posterior & 8 & $(19.5)$ \\
Concomitante & 6 & $(14.6)$ \\
\hline $\begin{array}{l}\text { CUADRO no. } 5 \\
\text { Relación en tiempo entre la alteración severa } \\
\text { lel sueño y la convulsión. }\end{array}$ \\
\hline
\end{tabular}

no permite sacar conclusiones sobre la acción de las drogas en el Trastorno del Sueño se podría especular sobre si el problema pudiera estar determinado por una alteración de la electrogénesis cerebral sin localización definida por el momento; el porcentaje alto de respuestas moderadas o maleas para indicar también que además de la hipótesis anterior debe existir otra causa etiopatogénica del trastorno.

En el cuadro No. 7 se muestra la distribu-

\begin{tabular}{|l|r|}
\hline DROGA UTILIZADA & No. \\
\hline Barbitúrico & 46 \\
Tranquilizantes & 25 \\
Hindantoinato & 42 \\
Ninguno & 10 \\
& \\
RESULTADOS & \\
Excelente & 9 \\
Bueno & 17 \\
Moderado & 21 \\
Malo & 17 \\
\hline CUADRO No. 6 & \\
Tipo de tratamiento y resultado \\
terapeútico en relación con la \\
slteración severa del sueño. \\
\hline
\end{tabular}

ción de los resultados EEG de acuerdo a la presencia a ausencia de convulsiones. Se puede observar que el porcentaje de EEg,s normales en el grupo con convulsiones es similar al descrito en otros trabajos; en el grupo sin convulsiones - si se descuentan los noños a los que no se le pidio EEG - la proporción es sensiblemente igual, lo cual está en favor de la organicidad como causante del trastorno.

En el cuadro No. 8 la distribución porcen- 


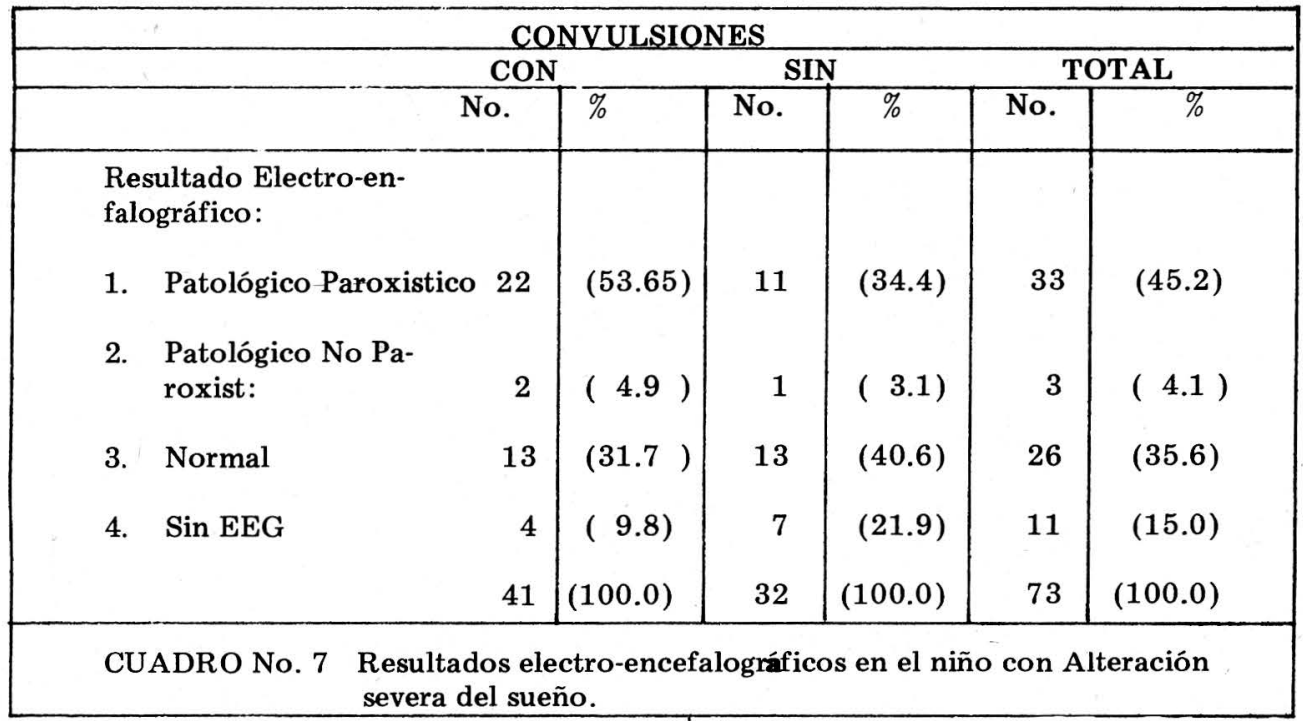

\begin{tabular}{|c|c|c|}
\hline TIPO DE COMPORTAMIENTO & No. & $\%$ \\
\hline 1. Normal & 20 & $(27.4)$ \\
\hline 2. Anormal & 53 & $(72.6)$ \\
\hline \multicolumn{3}{|l|}{ TIPO DE ALTERACION } \\
\hline 1. Hiperquinesia & 29 & $(54.7)$ \\
\hline 2. Irritabilidad exagerada & 37 & (69.8) \\
\hline $\begin{array}{l}\text { 3. Nerviosismo, susto y llanto } \\
\text { facil, tímido, retraído etc. }\end{array}$ & 32 & $(60.4)$ \\
\hline Explosiones de agresividad & 21 & $(39.6)$ \\
\hline Breath holding spell & 18 & $(33.4)$ \\
\hline 6. Alteración del sueño persistente & 9 & $(17.0)$ \\
\hline 7. Enuresis & 4 & $(7.5)$ \\
\hline \multicolumn{3}{|l|}{ CUADRO No. 8} \\
\hline
\end{tabular}

- tual y las características de las alteraciones del comportamiento. Nuevamente se eonfirma que los síntomas y signos psicológicos también obedecen a problemas neurológicos sutiles y que estas manifestaciones también deben ser tratadas así como se trata el episodio convulsivo.

En el cuadro No. 9 se muestra la distribución y el porcentaje de alteraciones que comprometen el desarrollo psicomotor. Se confirma que el cerebro no es únicamente 


\begin{tabular}{|l|c|c|}
\hline \multicolumn{1}{|c|}{ DESARROLLO PSICO MOTOR } & No. & $\%$ \\
\hline 1. Normal & 49 & $(67.12)$ \\
2. Anormal & 24 & $(32.88)$ \\
TIPO DE ANORMALIDAD & 9 & $(37.5)$ \\
1. Retraso Psico-motor & 4 & $(16.6)$ \\
2. Parálisis Cerebral & 3 & $(12.5)$ \\
3. Retardo Mental & 14 & $(58.3)$ \\
4. Retraso Lenguaje & $\begin{array}{l}\text { Desarrollo Psico-motor en el niño con Alteración } \\
\text { CUADRO No. 9 }\end{array}$ \\
\hline
\end{tabular}

el mediador de actos sensorio motrices sino que interviene en todos los tipos de desarrollo humano y por lo tanto no debe ser excluido del análisis - al menos cuando se trata de alteraciones neuro o psicológicas del desarrollo infantil.

\section{RESUMEN}

Se hace un análisis de las alteraciones del Sueño en el niño con Alto Riesgo Perinatológico. Se sugiere que los trastornos que se registran en 74 NARP puede obedecer a una alteración eléctrica cerebral por la frecuencia con estos niños presen tan posteriormente episodios convulsivos y por la respuesta de algunos de ellos a las drogas anticonvulsivantes. También se sugiere una alteración bioquímica, posiblemente en el metabolismo de las monoaminas como lo sugieren otros autores y por la dramática respuesta de algunos niños a la administración de estimulantes corticales. La relación con el riesgo perinatológico es aparentemente tan obvia en nuestra serie que sugiere una relación causa-efecto en los Trastornos del Sueño.

\section{SUMMARY}

Sleep disorders study is presented, analyzing 73 cases of Hight Risk Perinatal babies. (HRPB).
The possible electric cerebral damage, splayned by frecuent convultions association, may be one of the etiological factors.

Otherwise, metabolism brain troubles of mono-amynas, by hipothetical mecanisms; are invoqued to splain favorable reactions with cortical pharmacological stymulation, in order to treat sleep disorders.

\section{REFERENCIAS}

1. Ditrichova J. et Al. Individual Differences in Infant's Sleep.

Dev Med. Child. Neurol 18, 182-188, 1976.

2. Delange $\mathrm{Mm}$ et $\mathrm{Al}$ : Les Diverses Stades du Sommeil cheaz le Nouveau Né et le Nourrisson. Revue Neurol. 107 271, 1961.

3. Bakwin - Bakwin - Chap 9 "Sleep". p. 63. Behavior Disorders in Children W. B. Saunders Co. Philadelphia 1963.

4. Bernal J.F. Night Waking in Infants during the First 14 Months.

Dev. Med. Child. Neurol 15 760, 1973.

5. Forsius H. The Finnish Skolt Lapp Children A. Child Psychiatric Study Acta Ped. Scand - Suppl 239. 1973.

6. Lenerd H.G. Sleep Studies in Infancy Acta Ped. Scand 59 572, 1970

7. Chao D.H.- Seizures in Infancy and Carly 
Childhood.

Med. Clin. N.A. 423991958

8. Schain R.J. - Neurology of Childhood Learning Disroders.

Williams \& Wilkings Co. Balto 1972.

9. Prechtl H.F.R. et Al: Organisation of Physiological parameters in normal and neurologically abnormal infants. Neuropädiatrie 1: 101, 1969.
10. Quevedo - C.J. Epilepsia Sine Epilepsia - Tribuna Médica No. 580 Junio 1974.

11. Quevedo - C,J. - Las Alteraciones del Sueño en el Diagnóstico Neuropsicológico. Tribuna Médica No. 1 Abril 1976.

12. Wender P.H. - Minimal Brain Dysfunction in Children Wiley Interscience - John Wiley \& Sons. 